\title{
ANÁLISE DA CARGA DE TRABALHO FÍSICO EM ATIVIDADES DE IMPLANTAÇÃO FLORESTAL EM ÁREAS DECLIVOSAS
}

\author{
Nilton César Fiedler ${ }^{1}$, Ronie Silva Juvanhol ${ }^{2}$, Elizabeth Neire da Silva Oliveira de Paula ${ }^{1}$, \\ Saulo Boldrini Gonçalves ${ }^{3}$, Flávio Cipriano de Assis do Carmo², Rômulo Mazieiro ${ }^{4}$ \\ ${ }^{1}$ Eng. Florestal, Dr., Depto. Engenharia Florestal, UFES, Jerônimo Monteiro, ES, Brasil - fiedler@ pq.cnpq.br; \\ elizabeth@cca.ufes.br \\ ${ }^{2}$ Eng. Florestal, Mestrando em Ciências Florestais, UFES, Jerônimo Monteiro, ES, Brasil - ronie_juvanhol.com; \\ flaviocipriano@hotmail.com \\ ${ }^{3}$ Eng. Florestal, Mestrando em Ciências Florestais, UNICENTRO, Irati, PR, Brasil - sauloboldrinig @ hotmail.com \\ ${ }^{4}$ Graduando em Engenharia Industrial Madeireira, UFES, Jerônimo Monteiro, ES, Brasil - mazieiro.ufes@ gmail.com
}

Recebido para publicação: 12/07/2011 - Aceito para publicação: 18/01/2012

\begin{abstract}
Resumo
Esta pesquisa teve o objetivo de avaliar a carga de trabalho físico em atividades de implantação florestal em áreas declivosas no sul do Espírito Santo e propor uma reorganização ergonômica para melhoria dos níveis de saúde, bem-estar e satisfação dos trabalhadores. A carga de trabalho físico foi avaliada por intermédio do levantamento da frequência cardíaca, com o uso de monitores de frequência cardíaca nas atividades de construção de cercas, roçada manual, capina química, marcação de covas, coroamento, coveamento manual, enchimento de covas, adubação pré-plantio e plantio. A maior exigência física no trabalho foi verificada na atividade de coveamento manual (125 bpm), sendo esta classificada como moderadamente pesada. A menor exigência física no trabalho foi observada na atividade de adubação pré-plantio, com frequência cardíaca média de $87 \mathrm{bpm}$, sendo classificada como atividade leve. Foram verificadas diferenças significativas entre as atividades de implantação (médias da carga cardiovascular) pelo teste de Scott-Knott ao nível de 5\%, e a necessidade de reorganizar o ambiente de trabalho com inserção de pausas orientadas.

Palavras-chave: Ergonomia florestal; frequência cardíaca; plantio florestal; fisiologia do trabalho florestal.
\end{abstract}

\begin{abstract}
Analysis of the load of physical work in activities of forest implementation insteepareas. This research had the objective to evaluate the load of physical work in activities of forest implementation in steep areas in the south of the Espírito Santo, Brazil and to propose an ergonomic reorganization for improvement of the levels of health, well-being and satisfaction of the workers. The load of physical work was evaluated by the survey of the cardiac frequency with the use of monitors of cardiac frequency in the activities of construction of fencing, manual mowing, weeding chemical, marking of graves, weeding, manual pit, filling of pits, adubation pre-planting and planting. The biggest physical requirement in the work was verified in the activity of manual pit (125 bpm), being classified as moderately heavy activity. The lower physical demands at work were observed in adubation preplanting, frequently average cardiopath of $87 \mathrm{bpm}$, being classified as light activity. Significant differences had been verified enter the averages of the cardiovascular load and necessity to reorganize the environment of work with insertion of target pauses.

Keywords: Forestry ergonomics; cardiac frequency; forestry activities; physiology of forest work.
\end{abstract}

\section{INTRODUÇÃO}

Algumas atividades florestais de implantação, manutenção e colheita, dependendo da forma como são executadas, podem ser classificadas como extremamente pesadas, principalmente se realizadas de forma manual ou semimecanizada.

$\mathrm{Na}$ área florestal, as atividades de implantação são realizadas em ambientes abertos, onde normalmente os trabalhadores permanecem expostos às condições ambientais, muitas vezes desfavoráveis 
(VOSNIAK et al., 2010).

A maioria das áreas disponíveis para plantios florestais no sul do estado do Espírito Santo é de áreas acidentadas, nas quais não há possibilidade de se realizar as operações de forma mecanizada, o que traz como consequência aumento na demanda de mão de obra nas atividades florestais. Grande parte desta não recebe orientação e treinamento para adequar as atividades às condições de trabalho e condições ambientais satisfatórias. $\mathrm{O}$ deslocamento dos trabalhadores em áreas acidentadas, o alto desgaste físico das operações, o perigo de acidentes e o baixo índice de conforto térmico têm como consequência problemas de sobrecarga física e metabólica dos trabalhadores.

O esforço físico resulta de qualquer atividade desenvolvida pelo homem, seja durante o período de descanso até a prática de atividades física pesadas. O esforço mais intenso deve ser evitado, sob risco de consequências de danos físicos e psicológicos ao ser humano (SEIXAS, 1991). A avaliação da Carga de Trabalho Físico (CTF) foi o primeiro parâmetro tratado pela fisiologia do trabalho a fim de expressar a intensidade da atividade laboral posta ao indivíduo, cujo conhecimento é de grande aplicação na área da saúde do trabalhador (LOPES et al., 2011).

A aplicação de métodos fisiológicos visa permitir que a carga de trabalho se mantenha dentro de limites que tornem possível o trabalho em turnos de 8 horas (ORGANIZAÇÃO INTERNACIONAL DO TRABALHO (OIT), 1968).

A avaliação da carga física de trabalho foi o primeiro parâmetro tratado pela fisiologia do trabalho e continua sendo uma questão central para a maioria dos trabalhadores do mundo (FIEDLER $e t$ al., 2008). A frequência cardíaca é um importante indicador para avaliar a carga de trabalho, devido aos inúmeros conhecimentos adquiridos em fisiologia humana e à grande facilidade de registro dos dados (EDHOLM, 1968).

Determinado dispêndio energético pode causar diferentes exigências do sistema cardíaco, dependendo de condições como temperatura do ambiente, tipo de trabalho (estático ou dinâmico) e número de músculos envolvidos no trabalho dinâmico (SILVA, 1999; IIDA, 2005).

Partindo da hipótese de que as atividades de implantação florestal causam níveis diferenciados de carga cardiovascular nos trabalhadores, esta pesquisa teve como objetivo avaliar a carga física de trabalho exigido nas atividades de implantação florestal em áreas acidentadas no sul do Espírito Santo, visando à reorganização ergonômica e à melhoria dos níveis de segurança, saúde, bem-estar e satisfação dos trabalhadores florestais.

\section{MATERIAL E MÉTODOS}

A pesquisa foi desenvolvida durante a implantação da Floresta Piloto no Instituto Federal de Educação, Ciência e Tecnologia do Espírito Santo (IFES), localizado no município de Alegre, ES, em coordenadas geográficas $20^{\circ} 46^{\prime} 10^{\prime \prime}$ de latitude Sul e $41^{\circ} 27^{\prime} 33^{\prime \prime}$ de longitude Oeste de Greenwich, com altitude média de 254 metros. Avaliou-se 45 trabalhadores durante os meses de novembro de 2010 a março de 2011, em uma área de 52,7 hectares (Figura 1). A pesquisa foi realizada durante a implantação dos experimentos com mudas de espécies nativas acondicionadas em sacolas plásticas $(20 \mathrm{~cm}$ de altura por $8 \mathrm{~cm}$ de largura). O clima da região é classificado como Cwa, segundo o sistema Köppen, apresentando chuvas no verão e seca no inverno, com precipitação média anual de $1200 \mathrm{~mm} \mathrm{e}$ temperatura média anual de $26{ }^{\circ} \mathrm{C}$ (SANTOS et al., 2009).

Os trabalhadores foram monitorados nas atividades de implantação florestal, compreendendo as operações de construção de cercas, roçada manual, capina química, marcação de covas, coroamento, coveamento manual, enchimento de covas, adubação pré-plantio e plantio. A descrição dessas atividades pode ser observada na tabela 1 .

Para o levantamento da frequência cardíaca dos trabalhadores, utilizaram-se monitores de frequência cardíaca, marca Polar CS 300, fixados à altura do peito dos trabalhadores, sendo os valores coletados pelo sensor em intervalos de 20 segundos, para representar a carga física do trabalhador de acordo com a atividade realizada. Paralelamente, realizou-se um estudo de tempos e movimentos, para dimensionar as atividades realizadas em função dos tempos consumidos.

O número mínimo de trabalhadores para representação de cada atividade foi estabelecido considerando-se um erro amostral máximo de $10 \%$, a partir de uma amostragem-piloto segundo a metodologia proposta por Conaw (1977), por meio da equação 1: 


$$
\mathrm{n} \geq \frac{\mathrm{t}^{2} * \mathrm{~S}^{2}}{\mathrm{e}^{2}}
$$

Em que: $\mathrm{n}=$ número de trabalhadores necessários;

$\mathrm{t}=$ valor tabelado a $10 \%$ de probabilidade (distribuição $\mathrm{t}$ de Student);

$\mathrm{s}=$ desvio-padrão da amostra;

e = erro admissível a $10 \%$ em função da média da atividade.

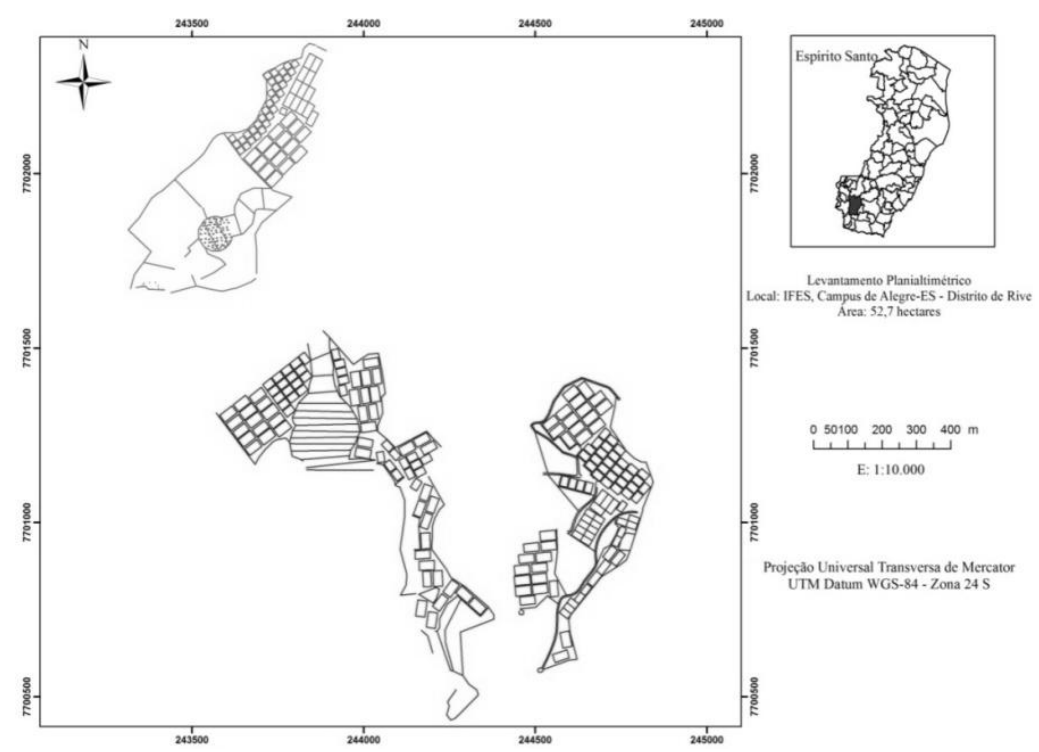

Figura 1. Mapa de localização da área da pesquisa.

Figure 1. Location map of study area.

Tabela 1. Descrição das atividades florestais avaliadas.

Table1. Description of forest operations analyzed.

\begin{tabular}{ll}
\hline Operações florestais & Caracterização da atividade \\
\hline Construção de cerca & $\begin{array}{l}\text { Estrutura composta por estacas de eucalipto tratado e arame farpado para } \\
\text { delimitação das áreas e prevenir ou restringir o acesso ao seu interior. }\end{array}$ \\
\hline Roçada manual & $\begin{array}{l}\text { Uso da foice para corte da vegetação arbustiva rasteira (controle de invasoras, } \\
\text { predominantemente de Brachiaria sp.). }\end{array}$ \\
\hline Capina química & $\begin{array}{l}\text { Aplicação de herbicidas para eliminar invasoras. Feito de forma manual, com o } \\
\text { pulverizador costal. }\end{array}$ \\
\hline Marcação de covas & $\begin{array}{l}\text { Determinação dos pontos onde deverão ser abertas as covas. Uso de barbante e } \\
\text { bambu com medidas padronizadas. }\end{array}$ \\
\hline Coroamento & $\begin{array}{l}\text { Eliminação de invasoras ao redor da muda (círculo). Atividade realizada de } \\
\text { forma manual, com enxada (raio 60 cm). }\end{array}$ \\
\hline Coveamento manual & $\begin{array}{l}\text { Escavação do solo para o plantio na cova previamente demarcada, com auxílio } \\
\text { de enxadão. }\end{array}$ \\
\hline Enchimento de covas & Retorno de parte da terra da cova para o seu interior. Feito de forma manual. \\
\hline Adubação pré-plantio & Aplicação e mistura de adubo manualmente nas covas. \\
\hline Plantio & Retirada do saco plástico e introdução das mudas na cova manualmente. \\
\hline Fonte: Adaptado de Pereira, 2010. &
\end{tabular}

De posse dos dados, determinou-se a carga cardiovascular no trabalho, que corresponde à percentagem da frequência cardíaca média do trabalho em relação à frequência cardíaca máxima tolerável para uma jornada de trabalho de 8 horas diárias, não devendo ultrapassar $40 \%$ da frequência cardíaca do trabalho. Para o cálculo, utilizou-se a metodologia proposta por Apud (1989), obtida por meio da equação 2:

$$
C C V=\frac{(\mathrm{FCT}-\mathrm{FCR})}{(\mathrm{FCM}-\mathrm{FCR})} \times 100
$$


Em que: $\mathrm{CCV}=$ carga cardiovascular, em \%;

$\mathrm{FCT}=$ frequência cardiovascular de trabalho, em bpm (batimentos por minuto);

$\mathrm{FCR}$ = frequência cardíaca em repouso, em bpm;

$\mathrm{FCM}=$ frequência cardíaca máxima, em bpm.

A frequência cardíaca máxima é definida pela equação 3:

$$
\mathrm{FCM}=220-\text { idade }
$$

Em seguida, a carga cardiovascular foi comparada com o limite individual de capacidade cardiovascular de 40\%, sugerido por Apud (1989), pela equação 4:

$$
\mathrm{FCL}=0,40 \times(\mathrm{FCM}-\mathrm{FCR})+\mathrm{FCR}
$$

Em que: $\mathrm{FCL}$ = frequência cardíaca limite, em bpm (batimentos por minuto).

Para atividades que excederam a carga cardiovascular de $40 \%$ (acima da frequência cardíaca limite), visando reorganizar o trabalho na finalidade de um rendimento contínuo e evitar fadiga por sobrecarga física, determinou-se o tempo de repouso (pausa) necessário, segundo Apud (1989), pela equação 5:

$$
\operatorname{Tr}=\frac{\text { Ht }(\text { FCT }- \text { FCL })}{(\text { FCT }- \text { FCR })}
$$

Em que: $\mathrm{Tr}=$ tempo de repouso, descanso ou pausa, em minutos;

$\mathrm{Ht}=$ duração do trabalho em minutos.

Por fim, classificou-se a carga de trabalho de acordo com a metodologia proposta por Apud (1997), conforme tabela 2.

Tabela 2. Classificação da operação segundo a frequência cardíaca média no trabalho.

Table 2. Classification of the operation according to average cardiac frequency in the work.

\begin{tabular}{lc}
\hline Frequência cardíaca média & Classificação da atividade \\
\hline$<75$ & Muito leve \\
$75-99$ & Leve \\
$100-124$ & Moderadamente pesado \\
$125-150$ & Pesado \\
$>150$ & Extremamente pesado \\
\hline Fonte: Apud, 1997. &
\end{tabular}

Os dados de carga cardiovascular foram analisados por meio de um Delineamento Inteiramente Casualizado (DIC), constando de nove tratamentos e número variável de repetições (trabalhadores) conforme atividade realizada. A análise de variância foi aplicada. Quando diferenças estatísticas foram observadas, aplicou-se o teste de Scott-Knott, ao nível de 5\% de probabilidade.

\section{RESULTADOS E DISCUSSÃO}

Os resultados relativos ao número de trabalhadores pesquisados, desvio-padrão e número mínimo necessário por atividade são apresentados na tabela 3.

Como observado, as atividades avaliadas apresentaram o número de amostras superior em relação ao mínimo necessário, considerando-se um erro de $10 \%$ de probabilidade pelo teste t de Student.

Os resultados das análises relativas à carga física de trabalho e à carga cardiovascular por atividade são apresentados nas figuras 2 e 3 .

A atividade de maior carga física de trabalho em operações de implantação florestal nas áreas acidentadas foi o coveamento manual, enquanto a adubação pré-plantio foi a atividade de menor exigência física. 
Tabela 3. Atividades, número de trabalhadores avaliados, desvio-padrão e número mínimo de amostras necessárias por atividade pesquisada.

Table 3. Activities, number of evaluated workers, standard deviation and minimum number of samples necessary for activity searched.

\begin{tabular}{lccc}
\hline Atividades & $\mathbf{N}^{\mathbf{0}}$ de trabalhadores avaliados & Desvio-padrão & $\mathbf{n}^{*}$ \\
\hline Construção de cercas & 10 & 13,15 & 6,16 \\
Roçada manual & 15 & 15,02 & 4,99 \\
Capina química & 4 & 8,04 & 2,75 \\
Marcação de covas & 3 & 3,06 & 0,39 \\
Coroamento & 10 & 19,22 & 9,08 \\
Coveamento manual & 17 & 10,65 & 2,20 \\
Enchimento de covas & 3 & 3,00 & 0,32 \\
Adubação pré-plantio & 8 & 11,58 & 6,15 \\
Plantio & 10 & 11,22 & 4,88 \\
\hline *Número mínimo de trabalhadores & necessário para analisar cada atividade de implantação florestal ao nível de $10 \%$ de \\
probabilidade pelo teste t de Student. & & &
\end{tabular}

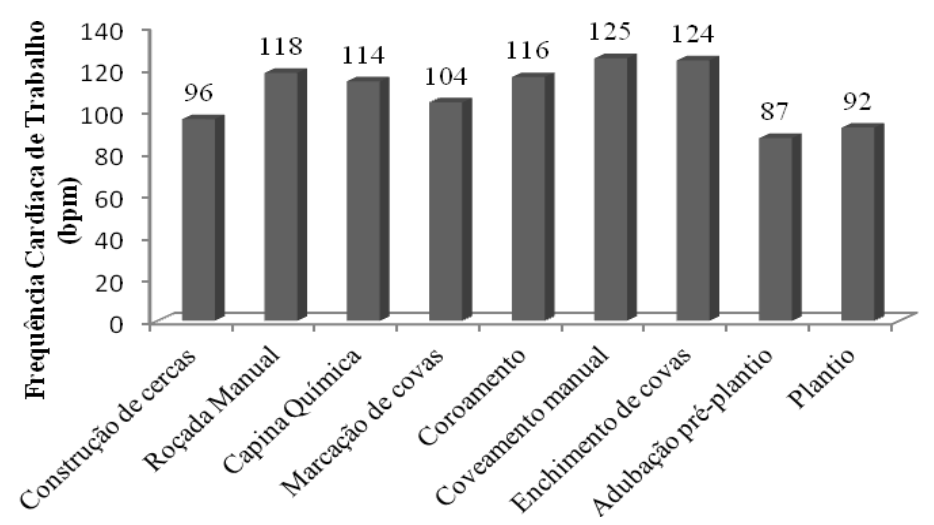

Figura 2. Representação da carga física média de trabalho nas operações de implantação florestal.

Figure 2. Representation of the average physical load at work implementation operations in forestry.

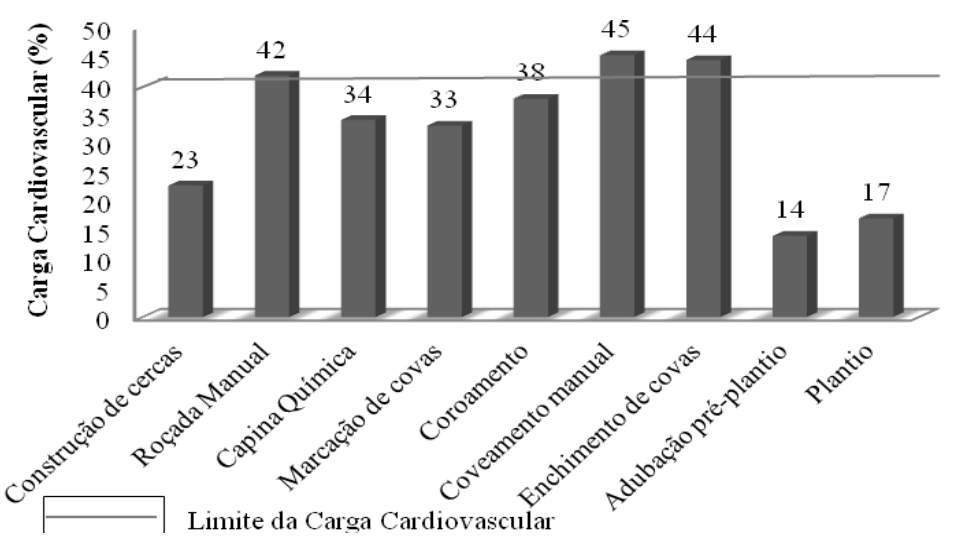

Figura 3. Representação da carga cardiovascular média de trabalho nas operações de implantação florestal.

Figure 3. Representation of the average cardiovascular load at work and cardiovascular load average implementation operations in forestry. 
Na tabela 4, são descritos os resultados por atividade analisada referentes à idade média dos trabalhadores, frequência cardíaca média no trabalho, frequência cardíaca média em repouso, frequência cardíaca máxima, carga cardiovascular, frequência cardíaca limite, tempo de repouso necessário e classificação do trabalho.

Tabela 4. Avaliação da carga física de trabalho nas operações de implantação florestal.

Table 4. Evaluation of the physical load at work in the implementation of forest operations.

\begin{tabular}{|c|c|c|c|c|c|c|c|c|}
\hline Atividade & $\begin{array}{l}\text { Idade } \\
\text { média } \\
\text { (anos) }\end{array}$ & $\begin{array}{c}\text { FCR } \\
(\mathbf{b p m})\end{array}$ & $\begin{array}{c}\text { FCT } \\
(\mathbf{b p m})\end{array}$ & $\begin{array}{l}\text { FCM } \\
(\text { bpm) }\end{array}$ & $\begin{array}{c}\text { CCV } \\
(\%)\end{array}$ & $\begin{array}{c}\text { FCL } \\
(\text { bpm) }\end{array}$ & $\begin{array}{c}\operatorname{Tr} \\
\text { (min/hora) }\end{array}$ & $\begin{array}{c}\text { Classificação da } \\
\text { atividade }\end{array}$ \\
\hline $\begin{array}{l}\text { Construção de } \\
\text { cercas }\end{array}$ & 36 & 70 & 96 & 184 & 23 & 116 & - & Leve \\
\hline Roçada manual & 34 & 70 & 118 & 186 & 42 & 116 & 1,90 & $\begin{array}{l}\text { Moderadamente } \\
\text { pesada }\end{array}$ \\
\hline Capina química & 28 & 74 & 114 & 192 & 34 & 121 & - & $\begin{array}{l}\text { Moderadamente } \\
\text { pesada }\end{array}$ \\
\hline $\begin{array}{l}\text { Marcação de } \\
\text { covas }\end{array}$ & 36 & 66 & 104 & 184 & 33 & 113 & - & $\begin{array}{l}\text { Moderadamente } \\
\text { pesada }\end{array}$ \\
\hline Coroamento & 31 & 71 & 116 & 191 & 38 & 119 & - & $\begin{array}{l}\text { Moderadamente } \\
\text { pesada }\end{array}$ \\
\hline $\begin{array}{l}\text { Coveamento } \\
\text { manual }\end{array}$ & 29 & 72 & 125 & 191 & 45 & 120 & 6,20 & Pesada \\
\hline $\begin{array}{l}\text { Enchimento de } \\
\text { covas }\end{array}$ & 31 & 73 & 124 & 189 & 44 & 119 & 5,36 & $\begin{array}{l}\text { Moderadamente } \\
\text { pesada }\end{array}$ \\
\hline $\begin{array}{l}\text { Adubação pré- } \\
\text { plantio }\end{array}$ & 37 & 71 & 87 & 183 & 14 & 116 & - & Leve \\
\hline Plantio & 31 & 72 & 92 & 189 & 17 & 119 & - & Leve \\
\hline
\end{tabular}

A atividade com maior exigência física de trabalho foi o coveamento manual, classificada como pesada, com frequência cardíaca média no trabalho de $125 \mathrm{bpm}$ e carga cardiovascular acima do limite máximo de $40 \%$. Para que a frequência cardíaca não ultrapasse o limite de $40 \%$, a frequência cardíaca no trabalho não deve ultrapassar o limite de $120 \mathrm{bpm}$. Para a atividade em questão, o limite máximo será alcançado com um repouso médio de 6,2 min./hora trabalhada (Tabela 4).

As atividades de enchimento de covas e roçada manual foram classificadas como moderadamente pesadas (Tabela 4), com frequência cardíaca média no trabalho de 124 bpm e 118 bpm, e carga cardiovascular de $44 \%$ e $42 \%$, respectivamente. Com base nos resultados, o trabalho deve ser reorganizado, promovendo um tempo de repouso médio de 5,36 min. por hora trabalhada, para a atividade de enchimento de covas, e 1,9 min. por hora trabalhada para a atividade de roçada manual, para que o limite máximo não ultrapasse 119 bpm e 116 bpm, respectivamente.

As atividades de menor frequência cardíaca média no trabalho foram a adubação pré-plantio, plantio e construção de cercas, classificadas como leve (Tabela 4), com frequência cardíaca média no trabalho de 87, 92 e 96 bpm, respectivamente. As demais atividades, embora classificadas como moderadamente pesadas, não tiveram o limite de carga cardiovascular ultrapassado, portanto poderão ser executadas normalmente, sem necessidade de reorganizar o ambiente de trabalho.

$\mathrm{Na}$ finalidade de estudar a distribuição por idade dos trabalhadores nas diferentes atividades, verificou-se que na principal atividade, que demanda conhecimento prévio, atenção e raciocínio (marcação de covas), estão inseridos trabalhadores experientes, com média de idade de 36 anos, o que possibilita maior rendimento na atividade e menor desgaste físico.

Nas atividades de maior exigência física, sugere-se trabalhadores com maior treinamento, menor idade e o uso de rodízios para um maior rendimento na operação. Dessa forma, evita-se fadiga por sobrecarga física ou mental durante a jornada de trabalho e, consequentemente, os acidentes. Com a adoção de tais medidas, é possível a melhoria das condições de saúde, segurança, bem-estar e satisfação dos trabalhadores.

A tabela 5 se refere aos testes de comparação entre médias das atividades pesquisadas, pelo teste de Scott-Knott a 5\% de probabilidade. 
Tabela 5. Teste de comparação entre médias, pelo teste de Scott-knott, a 5\% de probabilidade.

Table 5. Comparison test between averages by Scott-Knott test at 5\% probability.

\begin{tabular}{lccc}
\hline Tratamento & Repetição & CCV & Scott-Knott \\
\hline Coveamento manual & 17 & 45,15 & $\mathrm{~A}$ \\
Enchimento de covas & 3 & 44,33 & $\mathrm{~A}$ \\
Roçada manual & 15 & 41,63 & $\mathrm{~A}$ \\
Coroamento & 10 & 37,66 & $\mathrm{~B}$ \\
Capina química & 4 & 34,25 & $\mathrm{~B}$ \\
Marcação de covas & 3 & 32,49 & $\mathrm{~B}$ \\
Construção de cercas & 10 & 22,69 & $\mathrm{C}$ \\
Plantio & 10 & 17,02 & $\mathrm{C}$ \\
Adubação pré-plantio & 8 & 14,50 & $\mathrm{C}$ \\
\hline
\end{tabular}

Nota: CCV: carga cardiovascular.

O teste de comparação entre médias apontou diferença estatística entre médias da carga cardiovascular no trabalho, com as atividades de coveamento manual, enchimento de covas e roçada manual diferindo estatisticamente das atividades de coroamento, capina química e marcação de covas, que, por sua vez, diferiram da construção de cercas, plantio e adubação pré-plantio (Tabela 5). As atividades de maior carga cardiovascular e consequentemente de maior desgaste físico foram as atividades de coveamento manual, enchimento de covas e roçada manual.

\section{CONCLUSÕES}

- A implantação de florestas em áreas declivosas é constituída, em sua maioria, por atividades pesadas e moderadamente pesadas.

- A atividade de maior carga física de trabalho foi o coveamento manual, classificada como atividade pesada.

- As atividades de adubação pré-plantio e plantio foram classificadas como atividades de exigência física leve.

- As maiores cargas cardiovasculares no trabalho estiveram associadas às atividades de coveamento manual, enchimento de covas e roçada manual.

- Entende-se a necessidade de novos estudos, por se tratar de um estudo de caso, em que os fatores humanos e de ambiente irão variar em função de novas realidades. Esses novos estudos devem ser conduzidos visando à reorganização do ambiente de trabalho com vistas à melhoria de qualidade de vida dos trabalhadores e maior rendimento nas atividades de implantação florestal.

\section{AGRADECIMENTOS}

À Universidade Federal do Espírito Santo, pela oportunidade e estrutura física; à Fundação de Amparo à Pesquisa do Espírito Santo (FAPES), pelo apoio financeiro e concessão de bolsas de iniciação científica; ao LABCELF-UFES. Ao Governo do Estado do Espírito Santo, à Empresa Vale S.A. e ao Instituto Federal de Educação, Ciência e Tecnologia do Espírito Santo (IFES), Alegre, pelo apoio no desenvolvimento das pesquisas. Ao Eng. Florestal Emerson Espindula, pelo apoio de campo, e ao Eng. Agrimensor Pedro Quarto Júnior, pela confecção da planta de localização da área.

\section{REFERÊNCIAS}

APUD, E. Guidelines on ergonomics study in forestry. Genebra: ILO, 1989. 241 p.

Temas de ergonomia aplicados al aumento de la productividad de la mano de obra encosecha florestal. In: SIMPÓSIO BRASILEIRO SOBRE COLHEITA E TRANSPORTE FLORESTAL, 3, Vitória, 1997. Anais...Vitória: SIF/DEF, 1997, p. 46 - 60.

CONAW, P. L. Estatística. São Paulo: Edgard Blucher, 1977. 264 p. 
EDHOLM, O. G. Biologia do trabalho. Porto: Inova, 1968. 258 p.

FIEDLER, N. C.; FERREIRA, A. H. S.; VENTUROLI, F.; MINETTE, L. J. Avaliação da carga de trabalho físico exigido em operações de produção de mudas ornamentais no Distrito Federal - Estudo de Caso. Árvore, Viçosa, v. 31, n. 4, p. 703 - 708, 2007.

FIEDLER, N. C.; ALVES, R. T.; GUIMARÃES, P. P.; WANDERLEY, F. B. Análise da carga física de trabalho dos operadores em marcenarias no sul do Espírito Santo. Floresta, Curitiba, PR, v. 38, n. 3, 2008.

IIDA, I. Ergonomia: projeto e produção. São Paulo: Edgard Blucher, 2005. 465 p.

LOPES, E. S.; OLIVEIRA, F. M.; RODRIGUES, C. K. Determinação da carga física de trabalho na atividade de aplicação manual de herbicida. Ambiência, Guarapuava (PR) v. 7, n. 2 p. 329 - 337. 2011.

ORGANIZAÇÃO INTERNACIONAL DO TRABALHO (OIT). Guia de seguridad e hygiene em los trabajos forestales. Genebra, 1968. 244 p.

PEREIRA, D. P. Análise técnica e de custos de povoamentos de eucalipto sob preparo manual e mecanizado do solo em área declivosa no sul do Espírito Santo. 2010. 112 f. Dissertação (Mestrado em Ciências Florestais) - Programa de Pós-Graduação em Ciências Florestais, Universidade Federal do Espírito Santo, Jerônimo Monteiro, 2010.

SANTOS, L.; PASSOS, R.; CARDOSO, L.; SANTOS, C.; GARCIA, G. C.; CECÍLIO, R. Avaliação de atributos físicos de um Latossolo sob diferentes coberturas vegetais em Alegre (ES). Engenharia Ambiental: Pesquisa e Tecnologia, Brasília, DF, 2009.

SEIXAS, F. Avaliação do esforço físico dispendido em operações florestais: um exemplo na operação de colheita de sementes. Série Técnica IPEF, Piracicaba, v. 7, n. 22, p.1 - 16. 1991.

SILVA, K. R. Análise de fatores ergonômicos em marcenarias e do mobiliário do município de Viçosa - MG. 97 p. Dissertação (Mestrado em Ciência Florestal) - Universidade Federal de Viçosa, Viçosa, 1999.

VOSNIAK, J.; LOPES, E. S.; FIEDLER, N. C. Carga de trabalho físico e postura na atividade de coveamento semimecanizado em plantios florestais. Scientia Forestalis, Piracicaba, v. 28, n. 88, p. 589 598. 2010. 